\title{
On the characterisation of a Bragg spectrometer with X-rays from an ECR source
}

\author{
D. F. Anagnostopoulos ${ }^{\text {a }}$, S. Biri ${ }^{\text {b }}$, D. Gotta ${ }^{\text {c }}$, A. Gruber ${ }^{\mathrm{d}}$, \\ P. Indelicato ${ }^{\mathrm{e}}$, B. Leoni ${ }^{\mathrm{f}}$, H. Fuhrmann ${ }^{\mathrm{d}}$, L. M. Simons ${ }^{\mathrm{f}, *}$, \\ L. Stingelin ${ }^{\mathrm{f}}$, A. Wasser ${ }^{\mathrm{f}}$, J. Zmeskal ${ }^{\mathrm{d}}$ \\ ${ }^{a}$ Department of Material Science and Engineering, University of Ioannina, \\ Ioannina, Greece \\ ${ }^{\mathrm{b}}$ Institute of Nuclear Research (ATOMKI), Debrecen, P.O. Box 51, \\ H4001, Hungary \\ ${ }^{\mathrm{c}}$ Institut für Kernphysik, Forschungszentrum Jülich, D-52425 Jülich, Germany \\ ${ }^{\mathrm{d}}$ Institut für Mittelenergiephysik, Austrian Acad. of Sci., Vienna

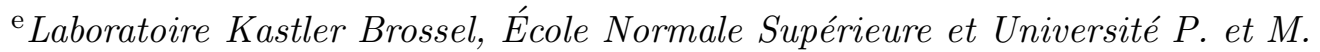 \\ Curie, Case 74, 4 place Jussieu, F-75252, Cedex 05, France \\ ${ }^{\mathrm{f}}$ Paul Scherrer Institut, Villigen PSI, CH5232 Villigen, Switzerland
}

\begin{abstract}
Narrow X-ray lines from helium-like argon emitted from a dedicated ECR source have been used to determine the response function of a Bragg crystal spectrometer equipped with large area spherically bent silicon (111) or quartz (101) crystals. The measured spectra are compared with simulated ones created by a ray-tracing code based on the expected theoretical crystal's rocking curve and the geometry of the experimental set-up.
\end{abstract}

Key words: X-ray spectroscopy, Exotic atoms, ECR source PACS: 29.30.Kv, 36.10.-k, 29.25.Ni

\footnotetext{
* Corresponding author. Address: L. M. Simons, WMHA/B24, CH 5232 Villigen, PSI

Email address: leopold.simons@psi.ch (L. M. Simons).
} 


\section{Introduction}

In an ongoing experiment at the Paul Scherrer Institute (Switzerland) the strong interaction broadening of the ground state level of pionic hydrogen of about $1 \mathrm{eV}$ should be determined with an accuracy of less than $10 \mathrm{meV}$ by measuring the line shape of X-ray transitions feeding the atomic 1s state $[1,2,3]$. The energies of the pionic hydrogen X-rays in question are 2.436, 2.886 and $3.043 \mathrm{keV}$ for the $2 \mathrm{p} \rightarrow 1 \mathrm{~s}, 3 \mathrm{p} \rightarrow 1 \mathrm{~s}$ and $4 \mathrm{p} \rightarrow 1 \mathrm{~s}$ transitions, respectively. The experiment uses a Bragg spectrometer in Johann mounting [4] and was equipped during recent measurements with spherically bent silicon or quartz crystals having a diameter of $100 \mathrm{~mm}$ and a thickness of $0.3 \mathrm{~mm}$ or $0.2 \mathrm{~mm}$, respectively. They were mounted by optical contact on glass lenses of ultimate quality. The curvature radius $R_{C}$ of the bent crystals is $R_{C}=2982.5 \pm 0.6 \mathrm{~mm}$. For these parameters bent crystal theory predicts a negligible influence of the bending process on the crystal's rocking curve [5]. Imperfections in the crystal material itself and defects produced during the fabrication could lead to deviations which should be determined experimentally. In order to meet the requirements imposed by the pionic hydrogen experiment a determination of the spectrometer's response function requires at least 20000 events in the calibration line. Moreover the measurement should be performed under conditions equivalent to the real experiment.

In earlier experiments a rough energy calibration was done by using X-rays from geometrically extended fluorescence targets excited by means of X-ray tubes [6]. A determination of the response function is out of reach with this method as the natural line widths are much broader than the resolution of the spectrometer. In addition the shape of these lines is distorted by satellite transitions.

Alternating the pionic hydrogen measurement in regular intervals with calibration experiments using narrow X-ray lines from other exotic atoms would be ideal. The measured low count rates of about 100/hour, however, render such an approach unfeasible.

The use of X-rays emitted from highly ionized ions therefore offers an appropiate solution albeit in a remote measurement. It makes use of the fact that for low to medium $\mathrm{Z}$ atoms the E1 X-rays from hydrogen-like ions as well as M1 X-rays from helium-like ions have natural line widths negligibly small compared to the expected resolution of the Bragg crystals. To give an example, the lifetime of the M1 transition of helium-like argon used mostly in the present study is $0.21 \mu \mathrm{s}$ [7]. The required X-rays are emitted in copious quantities from Electron Cyclotron Resonance (ECR) or Electron Beam Ion Trap (EBIT) sources. ECR sources are preferable as the X-rays are emitted

from a geometrically extended region important for a Johann set-up whereas 
EBIT sources produce X-rays in the region of the very compressed electron beam only, which would require an observation perpendicular to the electron beam cutting down the accepted intensity considerably. Decisive, however, is the low kinetic energy of the ions in ECR sources on the level of less than $1 \mathrm{eV}$ only, which had been determined at different ECR sources with high resolution optical spectroscopy $[8,9]$. From this a Doppler broadening of less than $40 \mathrm{meV}$ can be expected for transitions around $3 \mathrm{keV}$ energy in helium-like argon. In principle EBIT sources could be tuned to low kinetic energies of the captured ions by reducing the voltage of the drift tube, which, however, results in a drastic reduction in intensity [10].

The intention of this paper is much different from earlier publications using crystal spectrometers in combination with sources of highly charged ions. They dealt with a determination of plasma properties leading to a deeper understanding of the processes governing the physics of ECR sources $[11,12]$ or of Tokamak devices [13,14] as well as of plasma microsources [15]. At an EBIT source it became possible recently to determine even the polarization of x-ray emission lines with bent crystal spectrometry [16]. In the present paper, for the first time the characterisation of a crystal spectrometer with a narrow Xray line from an almost completely ionized atom is described. For this purpose the M1 transition from helium-like argon at $3.104 \mathrm{keV}(\lambda=3.994 \AA[7]$ close in energy to the $4 \mathrm{p} \rightarrow 1 \mathrm{~s}$ transition in pionic hydrogen was used.

\section{Experimental set-up}

The experiment as depicted in Figure 1 can be subdivided into three parts:

- The Electron Cyclotron Resonance Ion Trap (ECRIT) consists out of a superconducting split coil magnet, which together with special iron inserts, provides the mirror field configuration, an Advanced ECR source - Updated version (AECRU-U) style permanent hexapole magnet and a $6.4 \mathrm{GHz}$ power regulated microwave emitter [17]. The mirror field parameters provide one of the highest mirror ratios for ECR sources with a value of 4.3 over the length of the plasma chamber. The hexapole is cooled at the front pieces and on the inner radius by a forced flow of demineralised water. The plasma chamber is formed by a $0.4 \mathrm{~mm}$ thick stainless steel tube of inner diameter of $85 \mathrm{~mm}$ and a length of $265 \mathrm{~mm}$ axially limited by copper inserts. At the position of the hexapole gap the stainless steel tube is perforated by a series of $2.5 \mathrm{~mm}$ diameter holes allowing for radial pumping in addition to axial pumping. The microwave high frequency power is introduced directly to the plasma chamber with waveguides exhibiting a small angle to the axis. In this way the path of the X-rays was at no point cut by any obstacle which could distort the shape of the response function. 
An extraction voltage of $2 \mathrm{kV}$ had been routinely applied at the side opposite to the crystal spectrometer. The total ion current was measured as a control for a stable operation.

A reference pressure (without plasma) of $1.710^{-7}$ mbar was achieved. Gas filling was supplied radially by UHV precision leak valves through the gaps in the open structure hexapole. The gas composition was routinely surveyed with a quadrupole mass spectrometer. For an optimised plasma source a drastic increase of the number of energetic electrons was discovered which required the use of a cleaning magnet installed at a distance of one meter in front of the crystal.

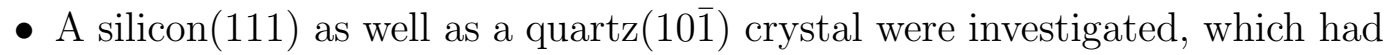
been recently applied for measuring pionic hydrogen transitions [20]. The Bragg angles $\Theta_{B}$, corresponding to the M1 transition of helium-like argon with an energy of $3.104 \mathrm{keV}$, are $\Theta_{B}=36.68^{\circ}$ for the quartz and $\Theta_{B}=39.57^{\circ}$ for the silicon crystal. The crystals were installed at a distance of $2330 \mathrm{~mm}$ from the centre of the ECRIT resulting in a position of the plasma about $500 \mathrm{~mm}$ outside the Rowland circle.

- A Charged Coupled Device (CCD) pixel detector with a pixel size of $40 \mu \mathrm{m}$ $\mathrm{x} 40 \mu \mathrm{m}$ and an energy resolution of $140 \mathrm{eV}$ at $3 \mathrm{keV}$ was used to detect the X-rays [21]. The detector consisted out of six chips with $600 \times 600$ pixels each resulting in a total height of $72 \mathrm{~mm}$ and a width of $48 \mathrm{~mm}$. The distance of the CCD detector from the crystal could be changed remotely over a length of $86 \mathrm{~mm}$ without breaking the vacuum.

The CCD detector and the associated electronics were protected against light as well as the high frequency stray field by a $30 \mu \mathrm{m}$ thick beryllium window installed in the vacuum tube in front of the CCD cryostat.

\section{Tuning the ECRIT}

In a first step with low high frequency power and minimum field values around $1.4 \mathrm{kG}$ the proper working of the whole set-up was checked with X-rays of low ionisation degree. In consequence the high frequency power was gradually increased and with the Bragg angles set to the proper values for a simultaneous observation of the well separable X-rays from both $\mathrm{Ar}^{10+}$ and $\mathrm{Ar}^{11+}$ an optimum for the high frequency power was found at about 450 Watt. The turn-around time from introducing a change of parameters to the evaluation of a measurement was about one minute. Going to Bragg angles valid for higher ionisation degrees the strength of the mirror field as well as the argon pressure were optimised for a maximum intensity of X-rays from highly excited ionised atoms. In addition a gas mixture $\mathrm{Ar} / \mathrm{O}_{2}$ with a mixing ratio of 1:9 was found to be necessary to achieve this goal [18]. The working pressure was $1.410^{-6}$ mbar. After a total tuning time of 10 hours the M1 transition ${ }^{3} S_{1} \rightarrow{ }^{1} S_{0}$ at 3104 
$\mathrm{eV}$ in $\mathrm{Ar}^{16+}$ could be observed as shown in Figure 2. The typical illumination time of the CCD chips of 1 min before readout resulted in a high probability of double hits per pixel even in the M1 peak. In order to reduce the intensity and especially in order to improve the peak/background ratio an aluminium collimator was inserted at a distance of $185 \mathrm{~mm}$ from the centre of the plasma into the direction of the crystal. It left a free hole with the dimensions 16 $\mathrm{mm}$ (horizontal) $\times 10 \mathrm{~mm}$ (vertical), which was wide enough not to influence the measurement of the response function.

A $6.4 \mathrm{GHz}$ emitter proved to be sufficient to provide a highly intensive source of M1 X-rays from $\mathrm{Ar}^{16+}$ ions in the special hybrid magnetic structure used with radial pumping together with the gas mixing technique. After turning the system on, it worked in a stable and reproducible way after about one hour warm up time. The observation of the X-rays alone is sufficient for a successful tuning in a short time thus making the usual momentum analysis of extracted ions superfluous. There was no measurable effect of the applied extraction voltage on the intensities of the X-rays for values between 0 and 6 $\mathrm{kV}$, which is the maximum achievable voltage.

\section{Response function of the crystal spectrometer}

With the ECR source a number of 20000 events was reached for the narrow M1 transition of helium-like Argon in about 30 minutes time to be compared with a number of 5000 counts reached after 40 hours with X-rays from pionic carbon formed when using methane gas. A total of about 10 hours was needed, however, to determine the spectrometer's response function in sufficient detail including changes of the distance CCD detector-crystal (focal scans) and changing apertures in front of the crystals. The measurements were confronted with theoretical predictions using the following procedure:

- The X-ray Oriented Program (XOP) program package [19] was used as a theoretical reference for the present measurements. It provided a theoretical rocking curve for a flat ideal crystal.

- The theoretical rocking curve was used as input for a Monte Carlo X-ray tracking routine. With it the Bragg reflection of a spherically bent crystal was calculated for the geometrical conditions of the special set-up parameters like source diameter, detector position and aperture at the crystal.

- The output of the tracking routine can be considered to be the theoretical limit of the response function which under ideal circumstances would reproduce the data. In order to allow for additional broadening this response function is folded with a Gaussian distribution. Its width was determined with a least square fitting of the data using the program package MINUIT from the CERN program library. The statistics of the Monte Carlo response 
function was chosen high enough not to contribute to the error of the fit.

Two types of changes in the experimental set-up were investigated with this procedure. At first spectra were taken for different distances of the CCD detector from the crystals (focal scans). In a second step with the CCD detector located at the focal position different apertures were mounted in front of the crystals.

The focal scans served two purposes:

- to verify the optimum focal position, which is given by $R_{C} \sin \Theta_{B}$. For this position the width of the response function should be at minimum. The minimum was searched for by moving the CCD in steps of 2-3 $\mathrm{mm}$ in direction to and from the crystal.

- to measure the line shape variations as a function of the crystal-detector distance which served as a check of the validity of X-ray tracking calculations.

An example of a spectrum far off focus is shown in Figure 3 for the quartz crystal with the CCD detector shifted by $12.5 \mathrm{~mm}$ from the focal position into the direction of the crystal. The FWHM of the transition is $657(7) \mathrm{meV}$ $(0.817(9) \mathrm{m} \AA)$ to be compared with the distribution at the focal position with a FWHM value of 459(6) meV (0.591(8) $\mathrm{m} \AA$ ) shown in Figure 4. The fit allowing for an additional Gaussian broadening reproduces the measured spectrum in detail.

In a second step the measured response function is compared with the simulated response function, as function of the crystal's reflection surface. This is achieved by applying apertures in front of the crystal and allows the study of possible irregularities in the response function due to crystal material imperfections and/or deviations from the spherical shape. Four different apertures were used: two circular ones with openings of diameter $40 \mathrm{~mm}$ and $60 \mathrm{~mm}$, respectively and two rectangular ones with horizontal openings of $40 \mathrm{~mm}$ and $60 \mathrm{~mm}$, respectively, and a free diameter of $95 \mathrm{~mm}$ elsewhere. In table 1 the results for the theoretical response function are confronted with the measurement. Both lineshapes were fitted with a single Gaussian and result in the values for the FWHM depicted in columns 2 and 3. The rocking curve width for a plane crystal, as obtained by XOP, fitted by a single Gaussian resulted in FWHM values of $106.6 \mu \mathrm{rad}$ for silicon and $97.4 \mu \mathrm{rad}$ for quartz.

The difference between the FWHM of the spectrometer's response function and the crystal's rocking curve reflects expected geometrical contributions (mainly due to the horizontal extension of the crystal-Johann broadening). In order to cope with the difference of the measured response function to the theoretical one, a least square fitting of the data was done with the width of a Gaussian folded with the theoretical response function as fit parameter. The 
result of this fit is shown in the fourth column and is considered to be the main result of the present investigation.

The Si crystal shows an additional Gaussian contribution of 34.1(1.8) $\mu \mathrm{rad}$ as an average of all apertures chosen. This can be interpreted as an overall deviation of the crystal geometry from spherical shape eventually caused by the production process or by a failure in the crystal material. For quartz the three smaller apertures show an additional broadening on average of about $24.2(1.8) \mu \mathrm{rad}$, but opening the aperture to $60 \mathrm{~mm}$ rectangular results in a considerable worsening. This points to an irregularity in the crystal material or its mounting in the upper and/or lower part of the reflecting surface and will be investigated in a future measurement for this special crystal.

The different dispersions of $3.756 \mathrm{meV} / \mu \mathrm{rad}$ for $\mathrm{Si}$ and $4.166 \mathrm{meV} / \mu \mathrm{rad}$ for quartz result in an energy resolution of about $478(6) \mathrm{meV}$ for the Si crystal and 506(8) $\mathrm{meV}$ for the quartz crystal, both equipped with a $60 \mathrm{~mm}$ horizontal aperture. Going to $60 \mathrm{~mm}$ circular aperture these values reduce to $456(8) \mathrm{meV}$ in the case of $\mathrm{Si}$ and to $459(6) \mathrm{meV}$ in the case of quartz.

The result of a measurement for a circular aperture with a free diameter of 60 $\mathrm{mm}$ is shown in Figure 4 for the quartz crystal together with the corresponding fit.

In terms of energy resolution the values for the additional Gaussian correspond to $100(8) \mathrm{meV}$ for quartz and to 128(6) meV for Si. Being different they cannot be caused by a common Doppler broadening caused by the motion of the argon ions. Moreover it should be recalled that from optical measurements an upper limit for the Doppler broadening of about $40 \mathrm{meV}$ had been obtained [8,9].

Compared to the total width of the response functions the Doppler broadening can be neglected and the present accuracy is sufficient for the determination of the strong interaction width in pionic hydrogen. As the response function has been measured at $3.104 \mathrm{keV}$ only, its shape has to be extrapolated to the lower energies of the different pionic hydrogen transitions. If one interpretes the additional Gaussian broadening as resulting from a geometrical deformation only this extrapolation is straightforward and the Gaussian broadening can be folded into the rocking curve before the X-ray tracking is performed.

\section{Conclusion and Outlook}

The characterisation of two large area spherically bent crystals was performed with M1 X-rays from helium-like argon with two orders of magnitude higher statistics than achievable with exotic atoms. The geometrical conditions of 
the characterisation were equivalent to the pionic hydrogen experiment. The measurement could be understood in terms of a Monte-Carlo X-ray tracking routine allowing, however, for an additional Gaussian broadening. ¿From this a prescription for extrapolating the result to different energies became possible.

In the future it is planned to improve on the peak/background ratio by insertion of a proper slit of tapered material with a hole of $1 \mathrm{~mm}$ (vertical) x 30 $\mathrm{mm}$ (horizontal) near the plasma chamber in the direction of the crystal.

In addition the achievable pressure will be optimised by reducing the surface of the iron insertion pieces drastically. The use of M1 transitions from helium-like chlorine ( supplied from the gaseous compound $\mathrm{CHClF}_{2}$, chlorodifluoromethane ) and helium-like sulfur from $\mathrm{SO}_{2}$ is envisaged for future experiments as a countercheck of the assumptions and conclusions made here. The energies at 2.430 and $2.756 \mathrm{keV}$, respectively, are close to the $2 \mathrm{p}-1 \mathrm{~s}$ and the $3 \mathrm{p}-1 \mathrm{~s}$ transition energies in pionic hydrogen.

Additionally a thorough investigation of X-ray intensities as a function of its radial origin inside the plasma is feasible with the special set-up of the crystal spectrometer. The described set-up allows to determine the Doppler broadening from the ion velocity distribution directly with crystals with better resolution. Similarly the observation of such a broadening as a function of the power and frequency injected into the ECR source may be studied.

\section{Acknowledgements}

The suggestions and the help of D. Hitz and K. Stiebing in the preparatory phase of the experiment are warmly acknowledged. We also thank H. Reist for offering the $6.4 \mathrm{GHz}$ emitter for free. Special thanks go to the Carl Zeiss Company in Oberkochen, Germany, which manufactured the Bragg crystals. Laboratoire Kastler Brossel is Unité Mixte de Recherche du CNRS n 8552.

\section{References}

[1] R-98.01, http://pihydrogen.web.psi.

[2] S. Biri, L. Simons, D. Hitz, Rev. Sci. Instrum. 71 (2000) 1116.

[3] D. F. Anagnostopoulos, S. Biri, V. Boisbourdain, M. Demeter, G. Borchert, J. P. Egger, H. Fuhrmann, D. Gotta, A. Gruber, M. Hennebach, P. Indelicato, Y. W. Liu, B. Manil, V. E. Markushin, N. Nelms, A. J. Rusi El Hassani, L. M.Simons, L. Stingelin, A. Wasser, A. Wells, J. Zmeskal, N.I.M. B205 (2003) 9 . 
[4] H. H. Johann, Zeitschrift f. Physik 69 (1931) 185.

[5] G. Hölzer, O. Werhahn, E. Förster, Cryst. Res. Technol. 33 (1998) 555.

[6] D. F. Anagnostopoulos, S. Biri, G. Borchert, W. H. Breunlich, M. Cargnelli, J. P. Egger, H. Fuhrmann, D. Gotta, M. Giersch, A. Gruber, M. Hennebach, P. Indelicato, T. S. Jensen, F. Kottmann, Y. W. Liu, B. Manil, V. E. Markushin, J. Marton, N. Nelms, G. C. Oades, G. Rasche, P. A. Schmelzbach, L. M. Simons, J. Zmeskal, Hyperfine Interactions 138 (2001) 131.

[7] A. M. Costa, M. C. Martins, F. Parente, J. P. Santos, P. Indelicato, At. Data Nucl. Data Tables 79 (2001) 223.

[8] C. Bernard, PhD thesis, Université J. Fourier, Lyon, 1996.

[9] N. Sadeghi, T. Nakano, D. J. Trevor, R. A. Gotscho, J. Appl. Phys. 70 (1991) 2552.

[10] M. R. Tarbutt, D. Crosby, E. G. Myers, N. Nakamura, S. Ohtani, J. D. Silver, Springer Lecture Notes in Physics 570 (2001)727.

[11] G. Douysset, H. Khodja, A. Girard, J. P. Briand, Phys. Rev. E61 (2000) 3015.

[12] P. Grübling, J. Hollandt, G. Ulm, Rev. Sci. Instrum. 69 (1998) 1367.

[13] O. Marchuk, G. Bertschinger, H.-J. Kunze, N. R. Badnell, S. Fritzsche, J. Phys. B: At. Mol. Opt. Phys. 37 (2004) 1951.

[14] M. Bitter, K. Hill, L. Roquemore, P. Beiersdorfer, D. Thorn, Ming Feng Gu, Rev. Sci. Instrum. 74 (2003) 1977.

[15] A. Ya. Faenov, S. A. Pikuz, A. I. Erko, B. A. Bryunetkin, V. M. Dyakin, G. V. Ivanenkov, A. R. Mingaleev, T. A. Pikuz, V. M. Romanova, T. A. Shelkovenko, Phys. Scripta 50 (1994) 333.

[16] D. L. Robbins, H. Chen, P. Beiersdorfer, A. Ya. Faenov, T. A. Pikuz, M. J. May, J. Dunn, A. J. Smith, Rev. Sci. Instrum. 75 (2004) 3717.

[17] Z. Q. Xie, Rev. Sci. Instrum. 69 (1998) 625.

[18] A. G. Drentje, A. Girard, D. Hitz, G. Melin, Rev. Sci. Instrum. 71 (2000) 623.

[19] M. Sanchez del Rio and R. J. Dejus. "XOP: Recent developments" SPIE proceedings (1998) 3448 .

[20] D. Gotta, M. Hennebach, Y. W. Liu, V E. Markushin, L. M. Simons, M. Cargnelli, H. Fuhrmann, M. Giersch, A. Gruber, A. Hirtl, J. Marton, J. Zmeskal, P. Indelicato, B. Manil, M. Trassinelli, D. F. Anagnostopoulos, N. Nelms, Phys. Scripta T 104 (2003) 94.

[21] N. Nelms, D. F. Anagnostopoulos, O. Ayranov, G. Borchert, J. P. Egger, D. Gotta, M. Hennebach, P. Indelicato, B. Leoni, Y. W. Liu, B. Manil, L. M. Simons, A. Wells, N.I.M. A484, (2002) 419. 


\section{Figures}

Fig. 1. Set up of the PSI ECRIT together with the Bragg crystal spectrometer

Fig. 2. The spectral region for argon $\mathrm{X}$-rays from $\mathrm{Ar}^{14+}, \mathrm{Ar}^{15+}$ and $\mathrm{Ar}^{16+}(\mathrm{M} 1$ transition) as emitted from the PSI ECRIT and measured with a Si (111) crystal. The energy values are from [7]. One channel (pixel) corresponds to $79 \mathrm{meV}$.

Fig. 3. The spectrum of the $\mathrm{Ar}^{16+} 3104 \mathrm{eV}$ M1 line measured with a quartz $(10 \overline{1})$ crystal is shown together with a fit for a detector position of $12.5 \mathrm{~mm}$ out of focus in direction of the crystal. A circular aperture with a diameter of $60 \mathrm{~mm}$ had been applied. One channel (pixel) corresponds to $93 \mathrm{meV}$.

Fig. 4. The spectrum of the $\mathrm{Ar}^{16+} 3104 \mathrm{eV}$ M1 line measured with a quartz $(10 \overline{1})$ crystal is shown for the focal position together with a fit. A circular aperture with a diameter of $60 \mathrm{~mm}$ had been applied. One channel (pixel) corresponds to $93 \mathrm{meV}$. 
Table 1

The FWHM in $\mu \mathrm{rad}$ of the theoretical response function is compared with the measurement for a Si (111) as well as a quartz (101) crystal for different openings (rectangular or circular) of the diffraction surface. The last column shows the FWHM for a Gaussian to be folded with the theoretical response function in order to fit the measurement best.

\begin{tabular}{llll}
\hline Crystal/aperture & Theor. resp. fct. & Measurement & Gaussian \\
\hline Si/Rect. 60 & $116.0(0.5)$ & $127.4(1.7)$ & $38.0(4.0)$ \\
Si/Circ. 60 & $114.5(0.5)$ & $121.5(2.2)$ & $30.3(3.5)$ \\
Si/Rect. 40 & $108.5(0.6)$ & $119.8(2.2)$ & $34.6(3.2)$ \\
Si/Circ. 40 & $108.7(0.6)$ & $117.5(2.1)$ & $34.2(2.7)$ \\
\hline quartz/Rect. 60 & $109.4(0.3)$ & $121.5(1.8)$ & $38.0(4.1)$ \\
quartz/Circ. 60 & $106.2(0.3)$ & $110.2(1.4)$ & $21.5(3.2)$ \\
quartz/Rect. 40 & $98.8(0.4)$ & $104.2(1.7)$ & $25.1(2.9)$ \\
quartz/Circ. 40 & $98.4(0.4)$ & $104.2(1.6)$ & $25.6(3.1)$ \\
\hline
\end{tabular}

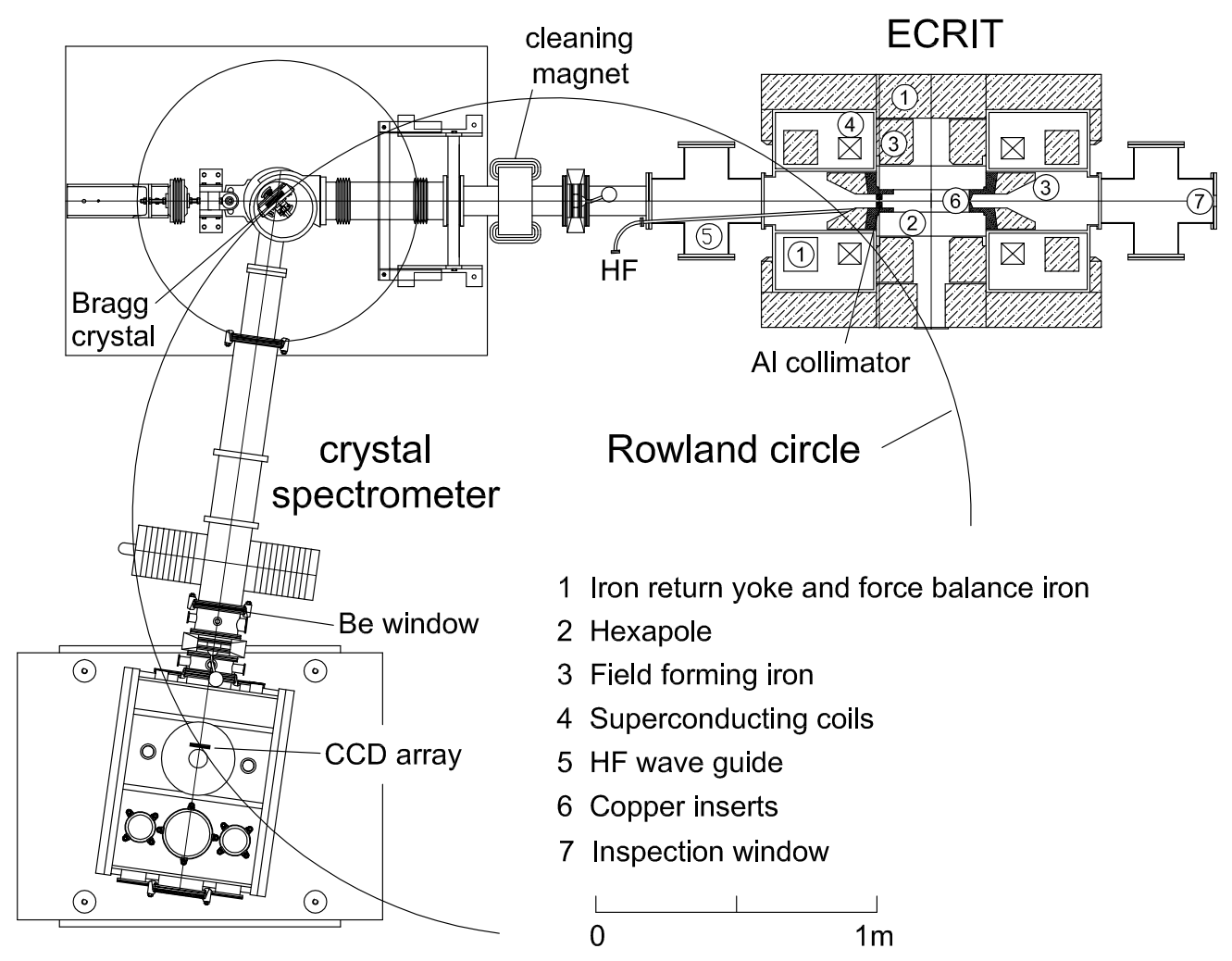

Fig. 1. Set up of the PSI ECRIT together with the Bragg crystal spectrometer 


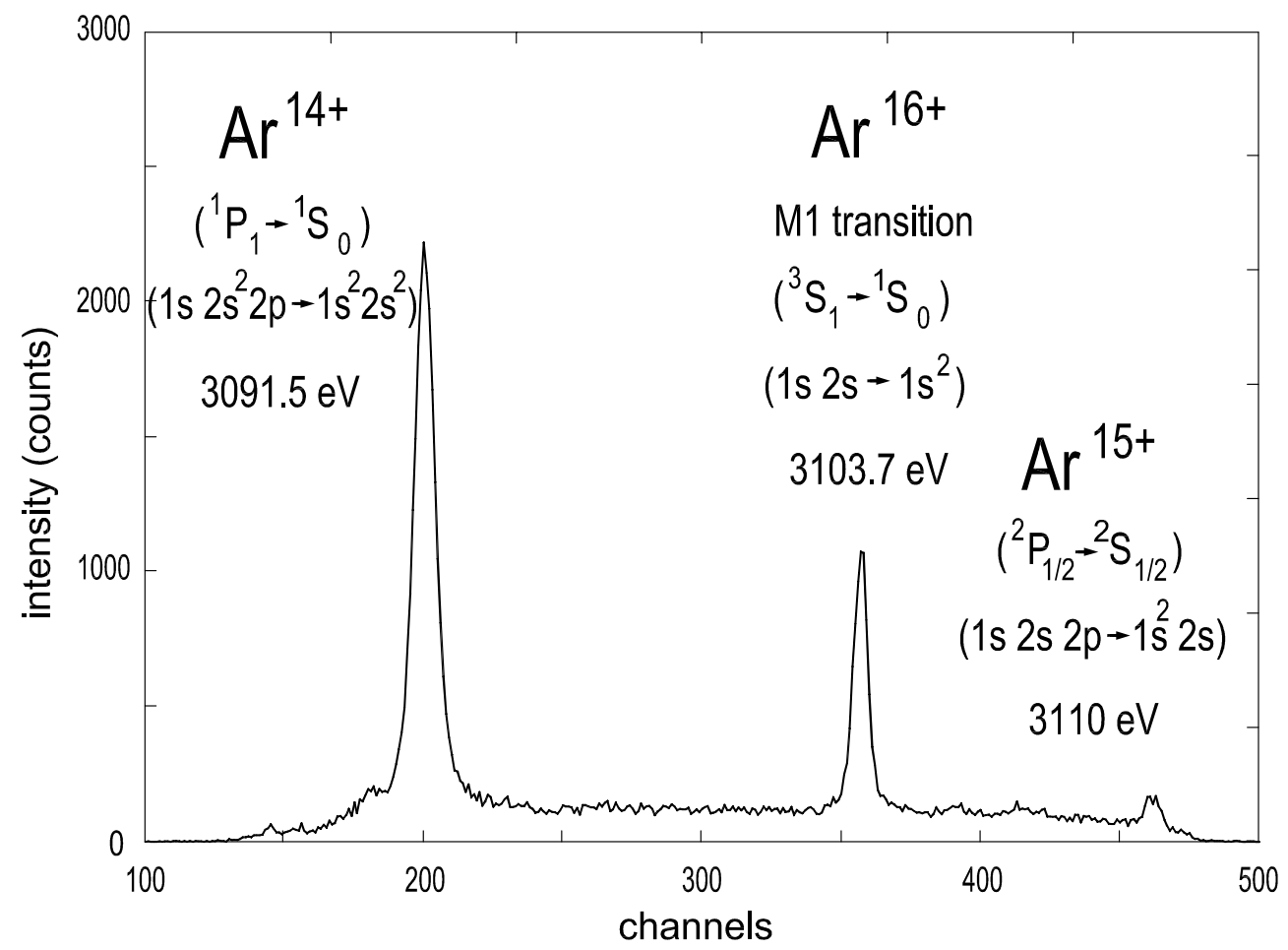

Fig. 2. The spectral region for argon $\mathrm{X}$-rays from $\mathrm{Ar}^{14+}, \mathrm{Ar}^{15+}$ and $\mathrm{Ar}^{16+}(\mathrm{M} 1$ transition) as emitted from the PSI ECRIT and measured with a Si (111) crystal. The energy values are from [7]. One channel (pixel) corresponds to $79 \mathrm{meV}$. 


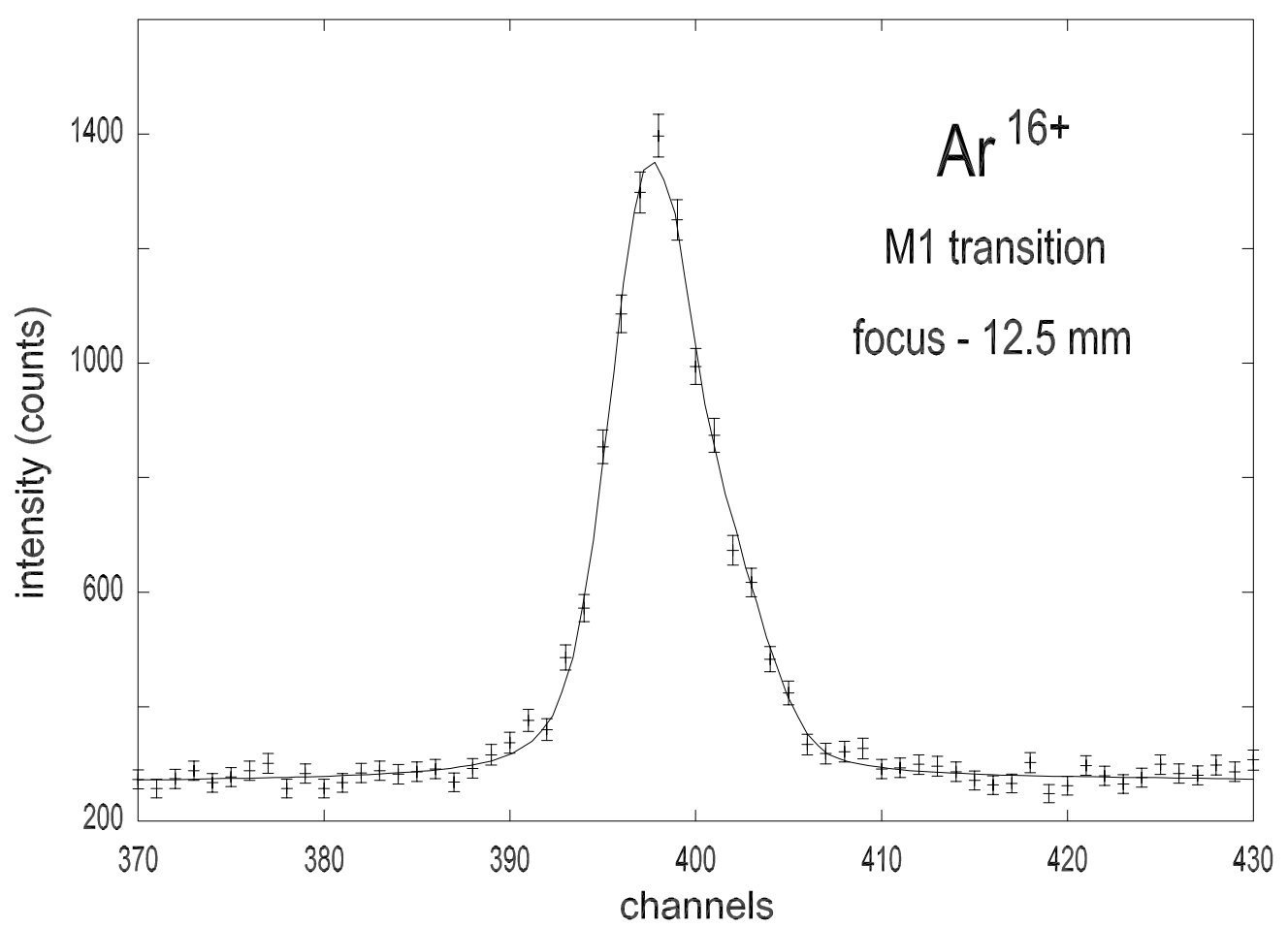

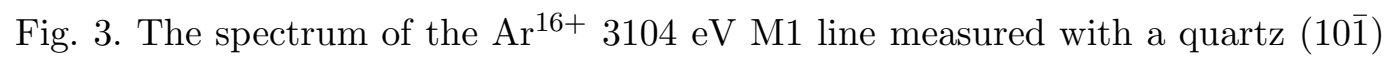
crystal is shown together with a fit for a detector position of $12.5 \mathrm{~mm}$ out of focus in direction of the crystal. A circular aperture with a diameter of $60 \mathrm{~mm}$ had been applied. One channel (pixel) corresponds to $93 \mathrm{meV}$. 


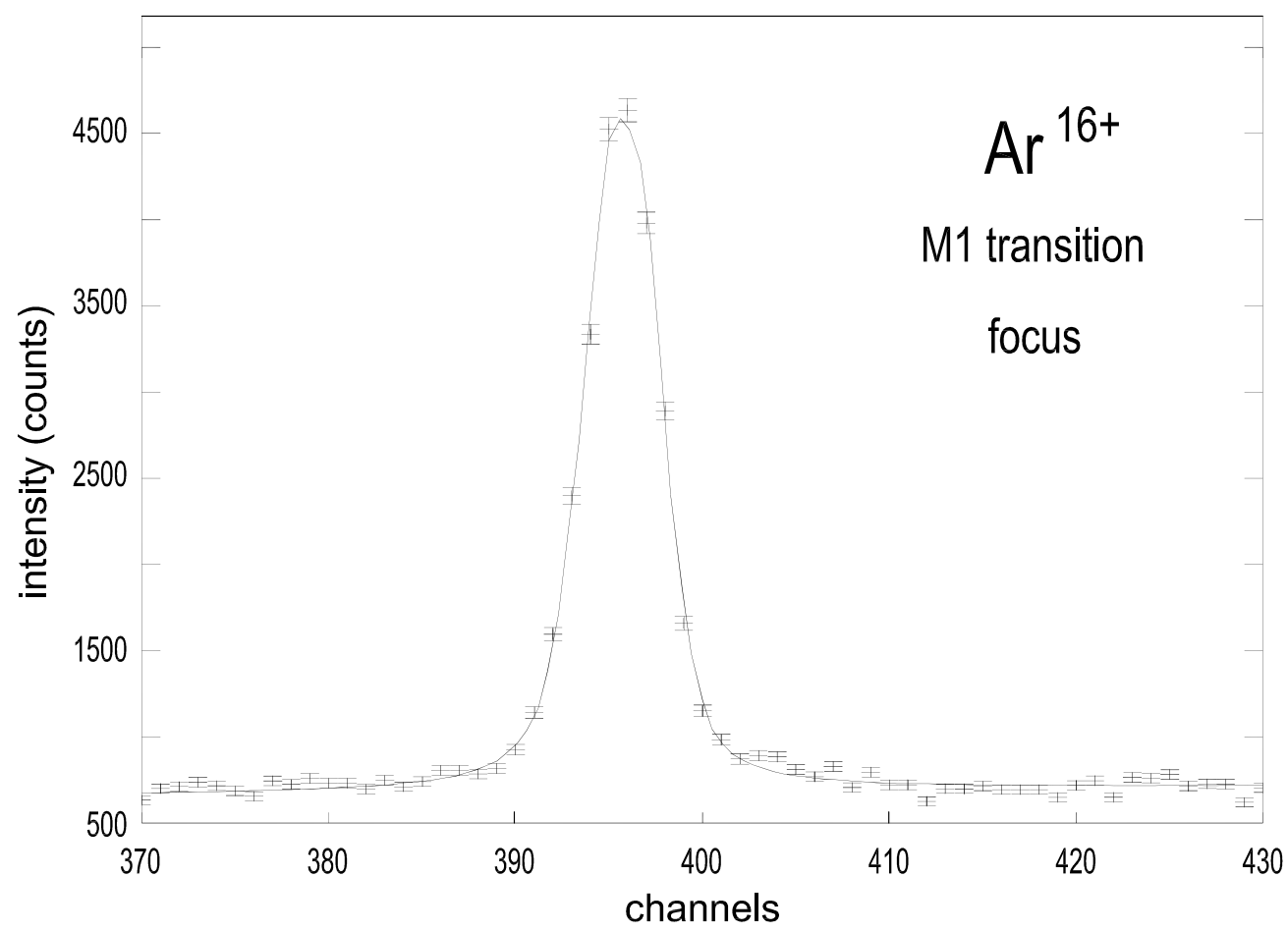

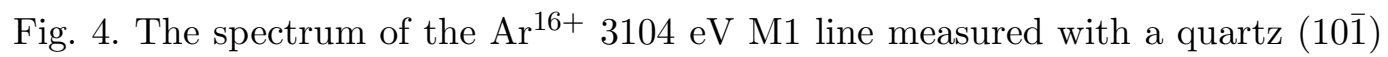
crystal is shown for the focal position together with a fit. A circular aperture with a diameter of $60 \mathrm{~mm}$ had been applied. One channel (pixel) corresponds to $93 \mathrm{meV}$. 